\title{
Development of Fire Scenarios for Car Parking Buildings using Risk Analysis
}

\author{
MOHD ZAHIRASRI MOHD TOHIR ${ }^{1}$ and MICHAEL SPEARPOINT ${ }^{1}$ \\ ${ }^{1}$ Department of Civil and Natural Resources Engineering \\ University of Canterbury \\ Christchurch 8140, New Zealand.
}

\begin{abstract}
This paper describes a relatively simple probability quantitative risk analysis model to determine appropriate fire scenarios for car parking buildings. The approach introduces a dimensionless measurement defined as fire risk level by multiplying probability by consequence. For the development of fire scenarios for car parking buildings, the key variables for the fire risk analysis are identified as vehicle parking distribution probability and how vehicles then form clusters of neighbours, vehicle classification, vehicle fire involvement probability, and the severity of vehicle fires. The selection of clusters of neighbouring vehicles and whether all vehicles in the cluster catch fire has the probability to affect the fire risk level. An example analysis is performed where a simple two-row, 100 space parking model with a $75 \%$ vehicle occupancy and 0.90 tendency factor weighting is used to obtain the vehicle distribution probability combined with various data sourced from the literature. It is found from the example analysis that fire risk level is largely driven by the vehicle fire involvement probability such that a single vehicle fire presents the worst case scenario in terms of fire risk.
\end{abstract}

KEYWORDS: risk, probablistic, car parking

\section{INTRODUCTION}

Vehicle parking buildings are commonly found in most modern urban environments. Such buildings can be stand-alone structures or attached to other occupancy types. The buildings can be multi-storey; above ground or below ground; be fully or partially enclosed; and be used to park a range of vehicle types (cars, vans, buses etc.). The usage characteristics of such buildings will depend on the service they provide: parking for patrons of a shopping mall, long-stay parking at an airport, parking for the residents of household units etc. This particular research is focussed on car parking buildings rather than for other vehicle types such as trucks or buses and the approach is similar to previous vehicle-fire related research [1], such that fire risk is equal to probability multiplied by consequence.

There have recently been several significant vehicle fires in car parking buildings and in some cases these have led to fatalities. For example, in 2006 seven fire fighters were killed in a fire in an underground car park in Gretchenbach, Switzerland [2]. Also in 2006 there was a car park fire in Bristol, United Kingdom where 22 vehicles were destroyed in the incident and one person died in the occupancy above the car park [2]. In terms of design Roosefid and Zhao [3] state that there are standard fire scenarios for car parking buildings required by the French authorities. The scenarios are seven cars including a utility vehicle in the same parking row, four cars including a utility vehicle situated in two adjacent parking rows and one car located at any position on the floor. These fire scenarios are applied so as to derive the most severe scenarios in terms of meeting fire resistance objectives. However, Roosefid and Zhao note that the greatest number of vehicles involved in a car parking fire was not more than three from incident statistics.

The life safety concerns of occupants and fire fighters and the appropriate design scenarios for structural design have led to consideration of the impacts of fires in car parking buildings. There is the need for further research into how to determine reasonable fire scenarios and raises the possibility that a single set of scenarios may not be applicable to all types of car parking buildings given the variations in design and use. The work presented in this paper is part of a larger risk-based research project where the first step is to create design scenarios which will be used for subsequent analysis. These scenarios need to consider the relative number, layout and type of vehicles that could be present in a parking building; the likelihood that multiple vehicles could burn simultaneously and the potential total energy that could be released by the burning vehicles. The focus in this paper is on a vehicle parking model that can identify the likelihood and magnitude of multiple vehicle clusters. 
Fire risk analysis is used to identify the impact of having a range of different vehicle fire scenarios in parking buildings. As a quantitative approach, the analysis establishes a dimensionless measurement for comparison, defined here as the fire risk level. For this research it is found that the probability component depends on a number of factors which are explained in the remainder of the paper. The consequence component is defined as the severity of the fire and is represented by the vehicle peak rate of heat release, and this is also discussed further in the paper. Clearly the most severe fire scenario does not necessarily have the highest risk level as it is compensated by the likelihood of the scenario occurring. Essentially the question becomes: for a given fire incident that starts in a specific vehicle what is the likely probability of a certain number of other vehicles being parked in neighbouring spaces, what are the likely types of vehicles in those spaces in terms of their combustible mass, will the fire spread to all of the neighbouring vehicles and what are the likely rate of heat release available from each vehicle that will contribute to the total heat release? Then how likely is this incident compared to the population of other similar incidents and which one of this population presents the greatest fire risk level?

The objective of this paper is to present an approach to establish vehicle parking scenarios using a probabilistic quantitative risk analysis method by incorporating a relatively simple vehicle parking model, statistical data on vehicle fleets, measurements of passenger vehicle heat release and vehicle fire incident data. The resulting risk analysis method could be used for the future specification of regulatory requirements for the design of car parking buildings but it has also been developed to be sufficiently flexible as such that it can benefit designers and regulators for the assessment of specific car parking buildings.

\section{FIRE RISK ANALYSIS}

In order to perform the fire risk analysis, the first step is to be able to understand the day-to-day situation in a parking building and then list all the key variables that are potentially associated with vehicle fires in the building. This approach follows the generalized concept for any fire risk analysis, i.e. to identify the hazards and then to quantify consequence and probability of those hazards [4].

The key variables identified are the vehicle parking distribution probability, i.e. the probability of vehicles being distributed in a particular pattern throughout the building at a given time; the vehicle classification i.e. the composition of different vehicle types in a fleet; the vehicle fire involvement, i.e. the likely number of vehicles involved in a fire using past incident data; and the severity of vehicle fires, where each of these variables is further explained in this paper. These variables are then used to create the necessary risk analysis components and the combination of these component variables determines a specific vehicle parking fire scenario.

Since the approach provides a numerical assessment, all of the key variables are quantitatively determined for each scenario. A probabilistic approach is used to demonstrate the severity of the fire as it relates to the likelihood of a given vehicle population and classification. The fire risk level is obtained by multiplying vehicle parking probability, vehicles classification, vehicle fire involvement probability and vehicle fire severity. Thus, this approach is used as a basis of a comparison to determine which scenario provides the highest fire risk.

Since there are almost limitless parking configurations; numbers of parking spaces; and parking space arrangements the approach used here attempts to be as generic as possible. Scenarios provide a general resemblance of the problem which can be related to most typical vehicle parking buildings. This generic approach is defines a simple two-row parking space arrangement as shown in Fig. 1 as a starting point for the research. For this approach, the number of parking spaces $n$ can be up to any desired value and the number of vehicles $x$ can be up to $n$ spaces. As an example, in Fig. 1, the value for parking spaces $n$ is 12 and the number of vehicles $x$ is 5 .

The potential for an open-ended level of depth for each component has led to the need to retain a consistent level of detail when deciding on how to obtain numerical inputs to the risk analysis. However even with the somewhat simplified approach described here, the calculations applied in the fire risk analysis are automated with the creation of a parking simulation model using Visual Basic for Applications in Microsoft Excel. 


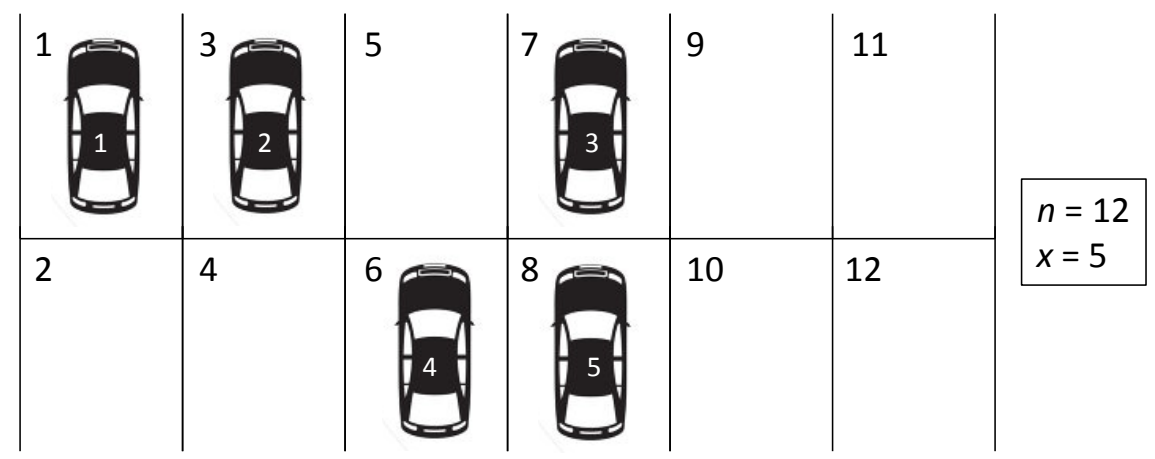

Fig. 1. Generic scenario.

\section{Vehicle parking probability}

The vehicle parking probability is used to determine the relative location of parked vehicles at a given time, i.e. the distribution of a given number of vehicles, $x$ across the available parking spaces, $n$. The distribution of vehicles is then used to identify clusters of neighbouring vehicles as discussed later. For this research the parking location distribution is managed by using a Monte Carlo approach. For example, considering the tworow model introduced in Fig 1, there are 12 parking spaces available for vehicle parking, however there are only 5 vehicles to fill the spaces. Each Monte Carlo run distributes the 5 vehicles into the 12 spaces randomly and therefore a particular scenario is formed. A successive application of the Monte Carlo method is then used to construct the foundation for the vehicle parking distribution input to the risk analysis.

In reality the distribution of parked vehicles is influenced by human behaviour factors. The study of these factors in the search for a parking space is interesting field of study as the topic itself is very broad. From the work by Waerden et al. [5], it is found that distance variables between parking spaces and other aspects (i.e. ticket machines, car park entrance, stairways and/or exit to final destinations) have an impact on parking space search behaviour. Thus a random approach to the Monte Carlo car placement is unlikely to resemble the reality of the parking distribution. The car placement procedure has been modified to include a parking tendency factor where it is assumed that vehicles tend to park at one end of the model to represent a distance variable. This parking tendency factor is governed by a user-defined weighting which controls the probability of vehicles being parked at one end of the model. The parking domain is equally split into a pair of two-row sections where a higher weighting results in a greater likelihood that a vehicle is placed in one of the pair over the other. As an example, this parking tendency factor can be visualized in Fig. 2 where a weighting of $80 \%$ is applied. In this example, the dotted lines represent the separation for the pair of two-row sections; Section 1 and Section 2 with Section 1 being nearer to the distance variable. A run of simulation will have 80 $\%$ chance of a car to be randomly placed in Section 1 while there is $20 \%$ chance of the car to be randomly placed in Section 2. This simplification, however, has its own limitations where the distributions within the sections are still random, thus if Section 1 is full, then vehicles in Section 2 will not be further affected by the distance variable. This can be improved by dividing the two-row parking model into many smaller sections although this more complicated algorithm has not been implemented in this work.

The work by Waerden et al. [5] showed three sets of parking data at a specific parking building in the Netherlands with a specific duration of time. These parking data consist of 4 two-row parking and 3 single-row parking spaces as well as two distance variables; a railway station and a passenger exit to a shopping mall, but for this analysis only the two-row parking data were used to match with the simple model proposed in this work. By assuming that the railway station is the dominant distance variable it can be inferred that the weighting of a tendency factor at peak times is around 0.90 while at off-peak times it is around 0.70 .

The parameters necessary for this component of the parking simulation model is the number of parking spaces, the number of vehicles, the number of iterations for the Monte Carlo simulations and the weighting for tendency factor. The input range for these variables are virtually unlimited, however, the limitations of Mi- 


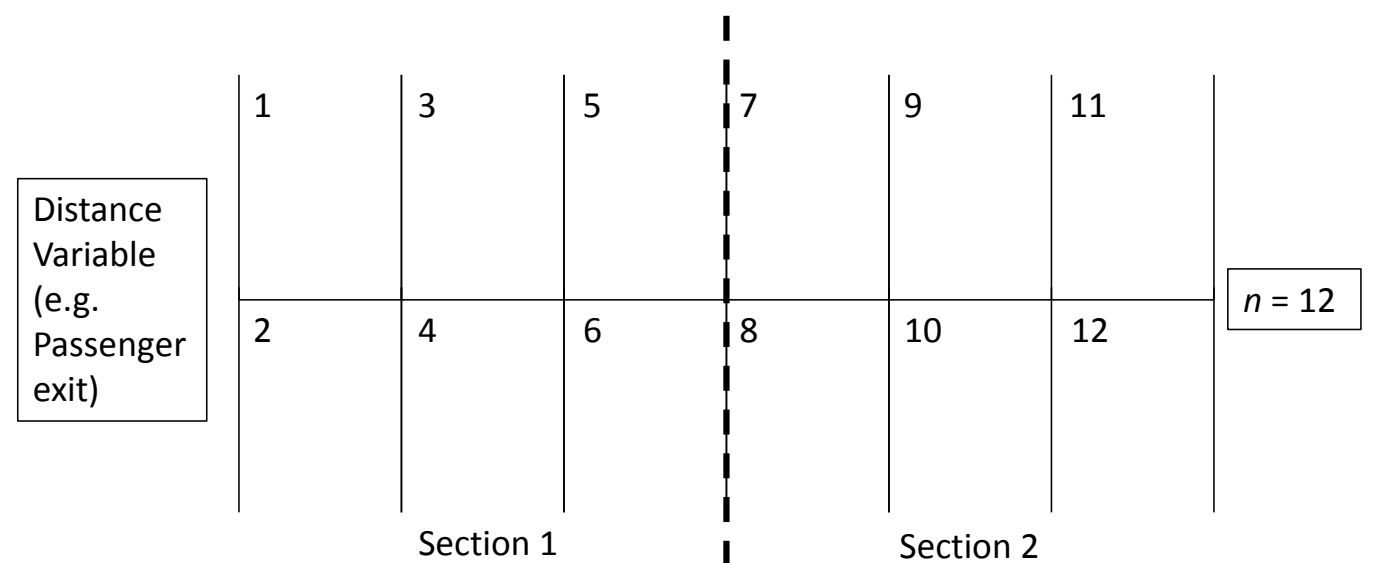

Fig. 2. Generic car parking scenario for 12 spaces with tendency factor.

crosoft Excel restricts the input up to certain maximum values. The output from the Monte Carlo simulations is the result for each iteration presented in an Excel spreadsheet for further analysis.

\section{Vehicle classification probability}

Since this research particularly focuses on car parking buildings, the scope of the study is limited to private road passenger vehicles. Current research by Tohir and Spearpoint [6] shows that there are numerous ways to categorise passenger vehicles and different jurisdictions have a variety of definitions for the purposes of classification. Some of the most common classifications are the vehicle engine size, the vehicle seating capacity, the vehicle dimensions (e.g. length, interior volume size), the vehicle curb weight, age, or wheelbase [7]. For this work, the American National Standards Institute (ANSI) [8] classification of road passenger vehicles based on curb weight of the vehicle is adopted (Table 1) as the mass is identified as a key parameter related to the potential fire load of vehicles.

Table 1. ANSI classification of vehicle by curb weight.

\begin{tabular}{|l|c|}
\hline \multicolumn{1}{|c|}{ Classification } & Curb weight \\
\hline Passenger car: Mini & $1500-1999 \mathrm{lbs}(680-906 \mathrm{~kg})$ \\
\hline Passenger car: Light & $2000-2499 \mathrm{lbs}(907-1134 \mathrm{~kg})$ \\
\hline Passenger car: Compact & $2500-2999 \mathrm{lbs}(1135-1360 \mathrm{~kg})$ \\
\hline Passenger car: Medium & $3000-3499 \mathrm{lbs}(1361-1587 \mathrm{~kg})$ \\
\hline Passenger car: Heavy & $\geq 3500 \mathrm{lbs}(\geq 1588 \mathrm{~kg})$ \\
\hline Van / MPV & Not defined \\
\hline SUV & Not defined \\
\hline
\end{tabular}

Following on from the selection of an appropriate vehicle classification system, associated statistics for the proportion of the road passenger vehicle types are presented to the fire risk level calculation. The proportion statistics are used by the parking model to select the classification of a vehicle applied to a simulation. The statistics for composition of this classification is obtained from data from the USA [9] and from the European Union [10] and is shown in Table 2.

\section{Vehicle fire involvement probability}

This component uses statistics from past vehicle fire incidents in car parking buildings as input into the fire risk analysis. Incident statistics are typically obtained from organizations that provide emergency fire fighting and rescue services where the nature of the details available depends the particular individual organization. In New Zealand these statistics are extracted from the New Zealand Fire Services (NZFS) fire incident reporting 
Table 2. Composition of vehicle classification.

\begin{tabular}{|l|c|}
\hline \multicolumn{1}{|c|}{ Classification } & Percentage composition \\
\hline Passenger car: Mini & $7 \%$ \\
\hline Passenger car: Light & $16 \%$ \\
\hline Passenger car: Compact & $20 \%$ \\
\hline Passenger car: Medium & $20 \%$ \\
\hline Passenger car: Heavy & $11 \%$ \\
\hline Van / MPV & $10 \%$ \\
\hline SUV & $16 \%$ \\
\hline
\end{tabular}

system (FIRS). For vehicle-related fires FIRS contains records for the date and time of incident, the incident type, the number of vehicles involved, the vehicle types, the vehicle year of manufacture, general property use, specific property use, location of origin, heat source, objects ignited, and fire cause.

However, these statistics often do not provide a high level of detail regarding the incident. For example it can be difficult to determine at what stage of the fire NZFS intervention took place or whether an automatic sprinkler system activated to suppress the fire. The statistics also do not state the total number of vehicles in the car park at the time of the fire or the relative parking space locations of the fire-affected cars and those not affected. It can therefore be hard to know whether a vehicle fire in a parking building had the potential to spread to other vehicles had it been allowed to continue unchecked.

Earlier research by Li and Spearpoint [11] shows that the probability of a vehicle catching fire in car parking buildings in New Zealand from 1995 - 2003 was $4.74 \times 10^{-6}$ per year. In this paper the analysis was extended using the same approach used by Li and Spearpoint up until 2012 using data from 2004 - 2012 obtained from the NZFS [12]. The probability for $2004-2012$ was $1.15 \times 10^{-6}$ per year, which is lower than the previous research making the overall probability from $1995-2012$ as $2.76 \times 10^{-6}$ per year. This probability is coupled with the vehicle fire involvement statistics to produce a vehicle fire involvement probability.

Vehicle fire involvement statistics have been obtained from the reported fire incidents in car park buildings acquired from the NZFS [12]. These statistics were strengthened by the collection of fire incident statistics in car park buildings compiled by Joyeux et al. [13] in 2002. The combined fire incident statistics is shown in Table 3. The table also shows the vehicle incidents probability and the annual vehicle fire involvement probability where the vehicle incidents probability is the number of incidents for a particular cluster divided by the total vehicle fire incidents and the vehicle involvement probability is the probability of a vehicle catching fire coupled with the vehicle incidents probability. Also from Table 3, there were a total of 401 incidents reported and the greatest number of vehicles involved were 7 with two incidents. The highest number of fire incidents are single vehicle cases with 344 incidents.

Table 3. Numbers of vehicles involved in fire and number of fire incidents.

\begin{tabular}{|c|c|c|c|}
\hline Number of vehicles involved & Number of incidents & Probability of incidents & $\begin{array}{l}\text { Vehicle fire involve- } \\
\text { ment } \\
\text { ity probabil- }\end{array}$ \\
\hline 1 & 344 & 0.858 & $2.37 \times 10^{-10}$ \\
\hline 2 & 27 & 0.067 & $1.86 \times 10^{-11}$ \\
\hline 3 & 21 & 0.052 & $1.45 \times 10^{-11}$ \\
\hline 4 & 4 & 0.010 & $2.75 \times 10^{-12}$ \\
\hline 5 & 3 & 0.007 & $2.06 \times 10^{-12}$ \\
\hline 6 & 0 & 0.000 & No data \\
\hline 7 & 2 & 0.005 & $1.38 \times 10^{-12}$ \\
\hline
\end{tabular}

Since the fire risk analysis requires data for up to maximum occupancy number of vehicles and the number of incidents only involves a maximum of 7 vehicles, a correlation for vehicle fire involvements against number of vehicles has been made. This correlation is used to predict the probability of a fire scenario occurring for
FIRE SAFETY SCIENCE-PROCEEDINGS OF THE ELEVENTH INTERNATIONAL SYMPOSIUM pp. 944-957 
higher numbers of vehicles than can be determined from the statistics. For this purpose, a simple correlation is obtained and shown in Fig. 3. A power law fit is used to correlate the known data because from the limited observation it is expected that the probability of incidents involving more vehicles will reduce. From this correlation an equation of $y=0.66 \times 10^{-6} x^{-2.67}$ where $x$ is the number of vehicles and $y$ is the probability of incidents is obtained. Thus, this equation is used to predict the vehicle fire incident probability for more than 7 vehicles.

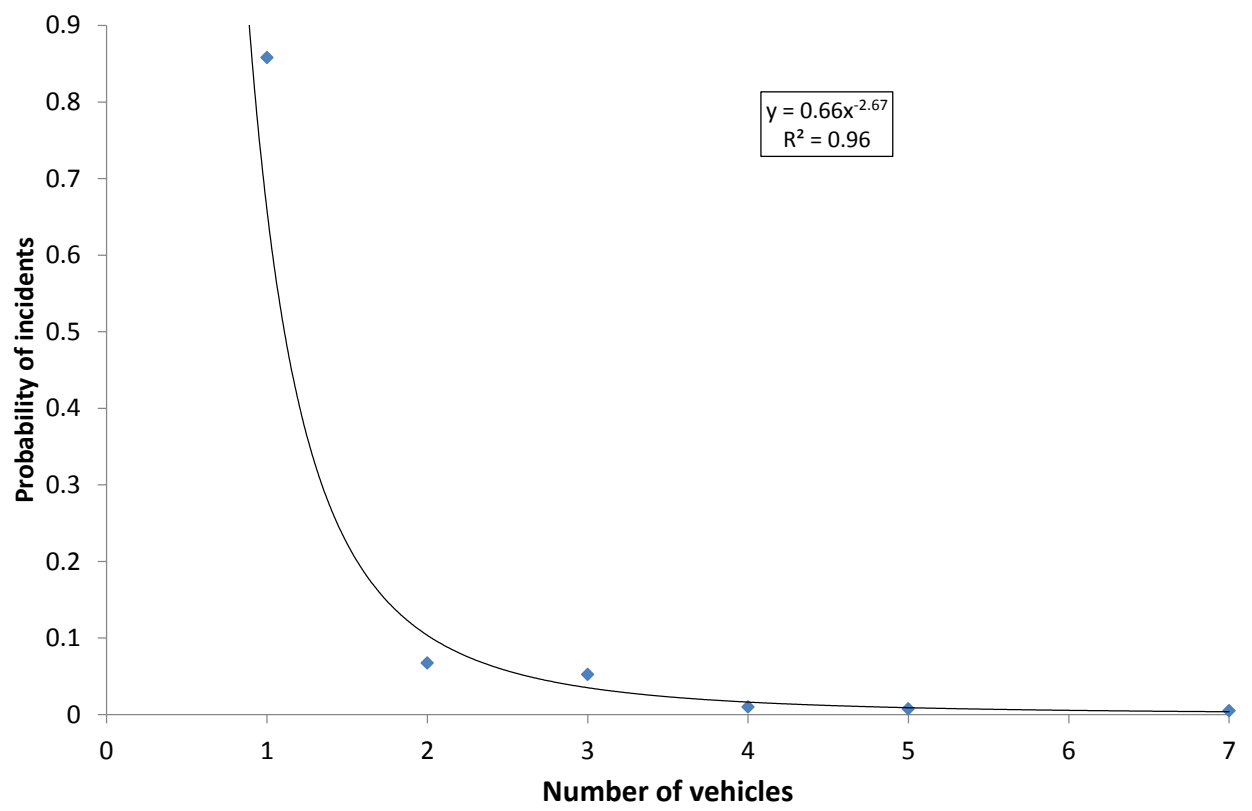

Fig. 3. Correlation of probability of incidents over the number of vehicles involved.

\section{Consequence}

For the consequence component of the risk analysis the heat release rate of road passenger vehicles is taken to be the critical parameter in that a higher heat release rate contributes to a higher fire risk level. The aforementioned research by Tohir and Spearpoint presents a distribution analysis for the fire severity characteristics of single passenger road vehicles using published heat release rate data [6]. The work collates full-scale laboratory experiment data from 41 single passenger road vehicles in the form of the peak rate of heat release, the time to reach peak rate of heat release and total heat released. Even though in that work only four classes were analysed i.e. Passenger Car: Mini, Light, Compact and Medium; the remaining classes can be estimated through the frequency data plot of the vehicle peak heat release rate against the vehicle curb weight.

Fig. 4 shows an example of the distribution plot of peak heat release for Passenger Car: Mini classification with the $5^{\text {th }}$ and $95^{\text {th }}$ percentile values indicated. A best-fit Weibull distribution has been determined for the data from 6 individual vehicle fire experiments. Average values for peak heat release rate are calculated for each classification that are then used for this study. The average values, $5^{\text {th }}$ and $95^{\text {th }}$ percentile distribution characteristics for each classification are shown in Table 4. However, due to limited data sets in the previously mentioned research work by Tohir and Spearpoint, the distribution characteristics for Passenger Car: Heavy, Van/MPV and SUV vehicle classification are extrapolated from the lower curb weight classifications.

It is noted that the procedures, standards and/or protocols varied between each experiment which likely lead to different effects on the fire spread, availability of air etc. and that the various heat release rate measurement techniques, namely mass loss rate, convective calorimetry and species-based calorimetry, could result in variability in the heat release rate measurements. However, due to limited data sets in each curb weight 


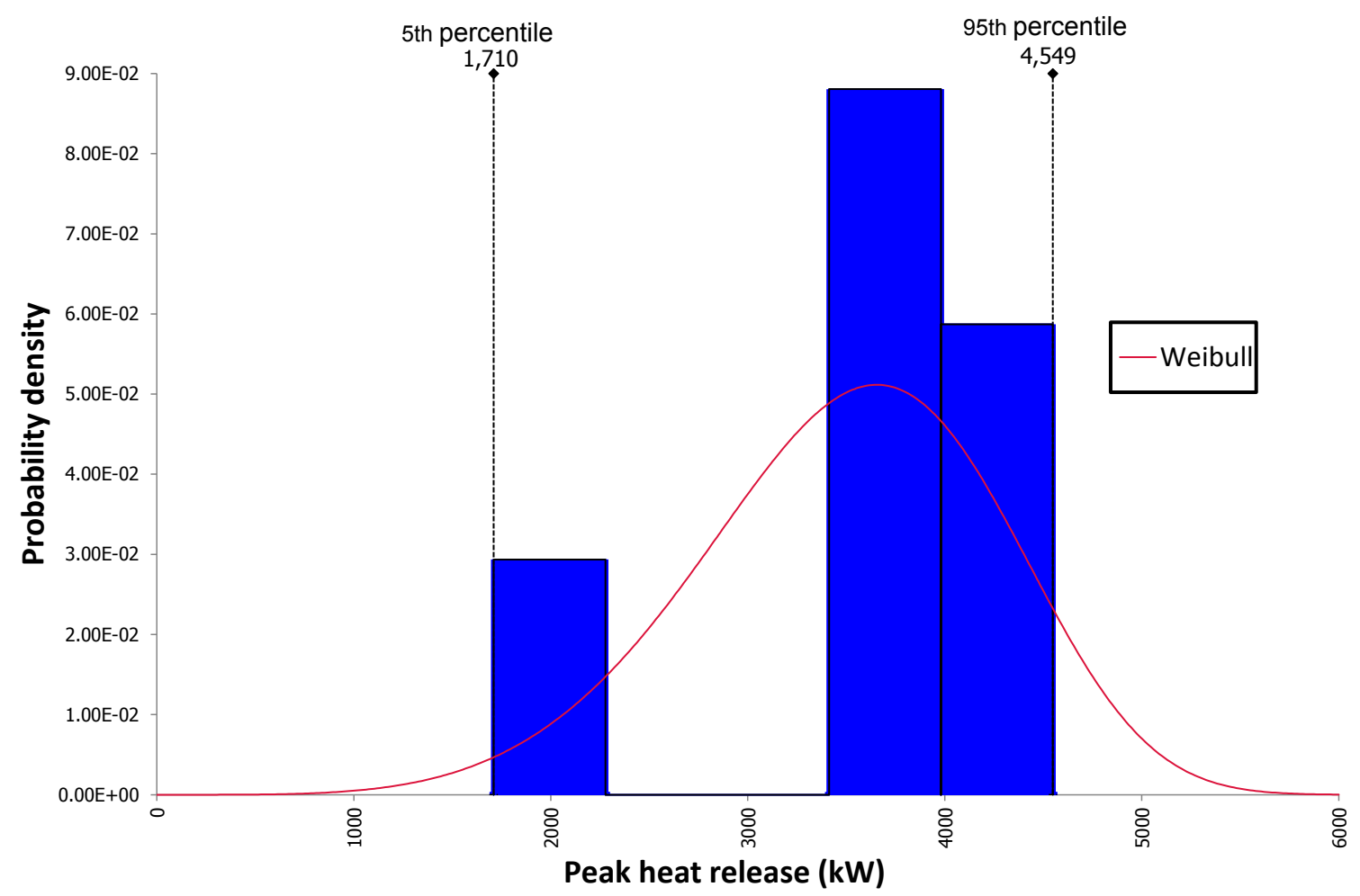

Fig. 4. Distribution plot of the vehicle peak heat release rate for Passenger Car: Mini.

Table 4. Average estimated peak heat release rate values with its distribution characteristics for each classification.

\begin{tabular}{|l|c|c|c|}
\hline \multicolumn{1}{|c|}{ Classification } & Average, $\mathbf{k W}$ & $\mathbf{5}^{\text {th }}$ percentile, $\mathbf{k W}$ & $\mathbf{9 5}^{\text {th }}$ percentile, $\mathbf{k W}$ \\
\hline Passenger car: Mini & 3490 & 1710 & 4549 \\
\hline Passenger car: Light & 4550 & 846 & 9802 \\
\hline Passenger car: Compact & 4150 & 1352 & 7406 \\
\hline Passenger car: Medium & 6890 & 3009 & 10850 \\
\hline Passenger car: Heavy & 8000 & 1849 & 13705 \\
\hline Van / MPV & 7000 & 1604 & 12016 \\
\hline SUV & 7000 & 1604 & 12016 \\
\hline
\end{tabular}

classification group meant it was not possible to create absolutely homogenous data sets that also provide sufficient items of data to be meaningful.

\section{APPLICATION OF THE RISK APPROACH}

\section{Cluster size assessment}

An example of the approach can be illustrated by presenting a simple parking problem. A single row of parking spaces is used for easier understanding of the process where the case of 5 parking spaces with 3 vehicles is illustrated. Fig. 5 shows all of the possible parking distribution scenarios for this case.

Two methods to determine the number of possible fire scenarios are described. For both methods the assumptions made are:

- $\quad$ Only full vehicle fire involvement is considered; either a vehicle has caught fire or it has not, there is no partial vehicle fire. 


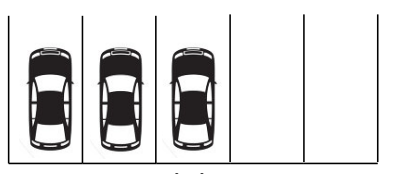

(a)

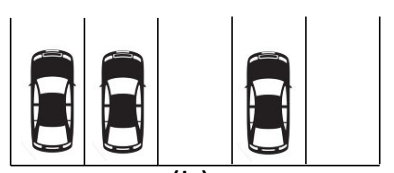

(b)

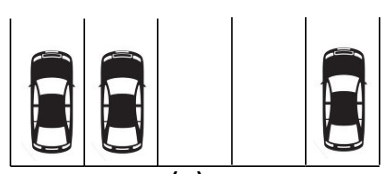

(c)

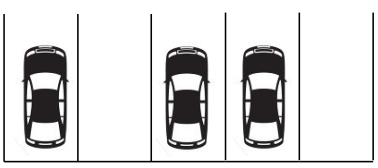

(d)

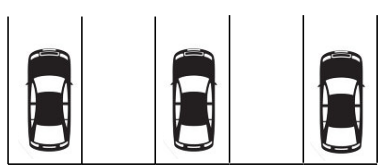

(e)

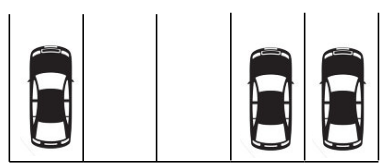

(f)

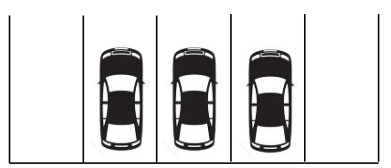

(g)

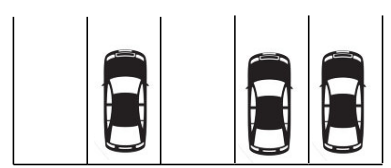

(h)

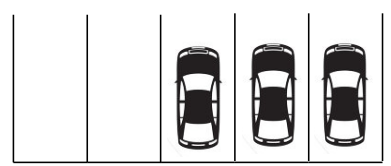

(i)

Fig. 5. All distribution scenarios for 5 parking spaces with 3 vehicles.

- There is no time dimension in the fire risk analysis, i.e. fires occur instantaneously and simultaneously.

For each vehicle on fire, a peak heat release rate is selected to maximise the risk.

- $\quad$ Fire spread does not occur across gaps formed by empty parking spaces.

The two methods are as follows:

i) Method 1 - In this method, vehicles located in contiguous parking spaces are considered to be a cluster such that they all catch fire simultaneously. Thus using Figure 5 distribution scenario (a), shows a cluster of three vehicles which means only a single fire scenario is present. However Figure 5 distribution scenario (b), shows a cluster of two vehicles parked next to each other and a separate cluster which consists of a single vehicle. Thus in distribution scenario (b) there are two clusters and therefore two possible fire scenarios.

ii) Method 2 - This method is an expansion on Method 1 in which each cluster can have all of the conceivable fire scenarios available to represent the possibility that the fire does not spread to neighbouring vehicles regardless of the size of fire. For example, in Figure 5 distribution scenario (a), there is one case of 3 vehicles catching fire, two cases of 2 vehicles catching fire and three cases of 1 vehicle catching fire. Therefore in total there are six possible fire scenarios within the single distribution scenario. For Figure 5 distribution scenario (b), there are two separate clusters but in terms of probable fire scenarios, there is one case of 2 vehicles catching fire and three cases of a single vehicle fire.

The calculation of the total possible number of fire scenarios and the associated probabilities for the example using the two methods is shown in Table 5. It is evident that the probability of one vehicle on fire using Method 1 is 0.50 whereas for Method 2 it is 0.66 which is a $16 \%$ difference. For two vehicles there is a 4 $\%$ difference and for 3 vehicles there is a $12 \%$ difference. These differences in probability show that using alternative assumptions for the possibility of fire occurring in multiple vehicles will result different outcomes in the fire risk level. For this research only Method 1 is adopted and further studied in detail although the possible implications of Method 2 are discussed.

\section{Maximum occupancy}

The starting point to determine the vehicle parking probability is to obtain data for parking occupancy values at different times and for car parking buildings that exhibit different usage characteristics. These data will FIRE SAFETY SCIENCE-PROCEEDINGS OF THE ELEVENTH INTERNATIONAL SYMPOSIUM pp. 944-957 
Table 5. Total possible fire scenarios and probability for both methods.

\begin{tabular}{|c|c|c|c|c|}
\hline & \multicolumn{2}{|c|}{ Method 1 } & \multicolumn{2}{c|}{ Method 2 } \\
\hline Number of vehicles & Frequency & Probability & Frequency & Probability \\
\hline 1 & 8 & 0.50 & 27 & 0.66 \\
\hline 2 & 5 & 0.31 & 11 & 0.27 \\
\hline 3 & 3 & 0.19 & 3 & 0.07 \\
\hline Total & 16 & 1.00 & 41 & 1.00 \\
\hline
\end{tabular}

present the parking trends for specific parking buildings. For this research, online data from several car parking buildings in San Francisco and Santa Monica, USA and two airport parking buildings in Switzerland and Italy have been obtained.

An example of this data is taken from the Sutter-Stockton Garage in San Francisco. This 24 hour car park provides parking spaces for nearby shops and offices. The parking data for this particular building is taken for a typical week from $5^{\text {th }}$ of May 2012 until $5^{\text {th }}$ of May 2013. Fig. 6 shows the normalized parking space occupancy as the number of vehicles parked over the total number of spaces available. Therefore the maximum occupancy is on Thursday where it almost reaches $75 \%$. This maximum occupancy provides a measure of the maximum exposed fire risk and thus a value of $75 \%$ is taken as starting point for the parking simulation model.

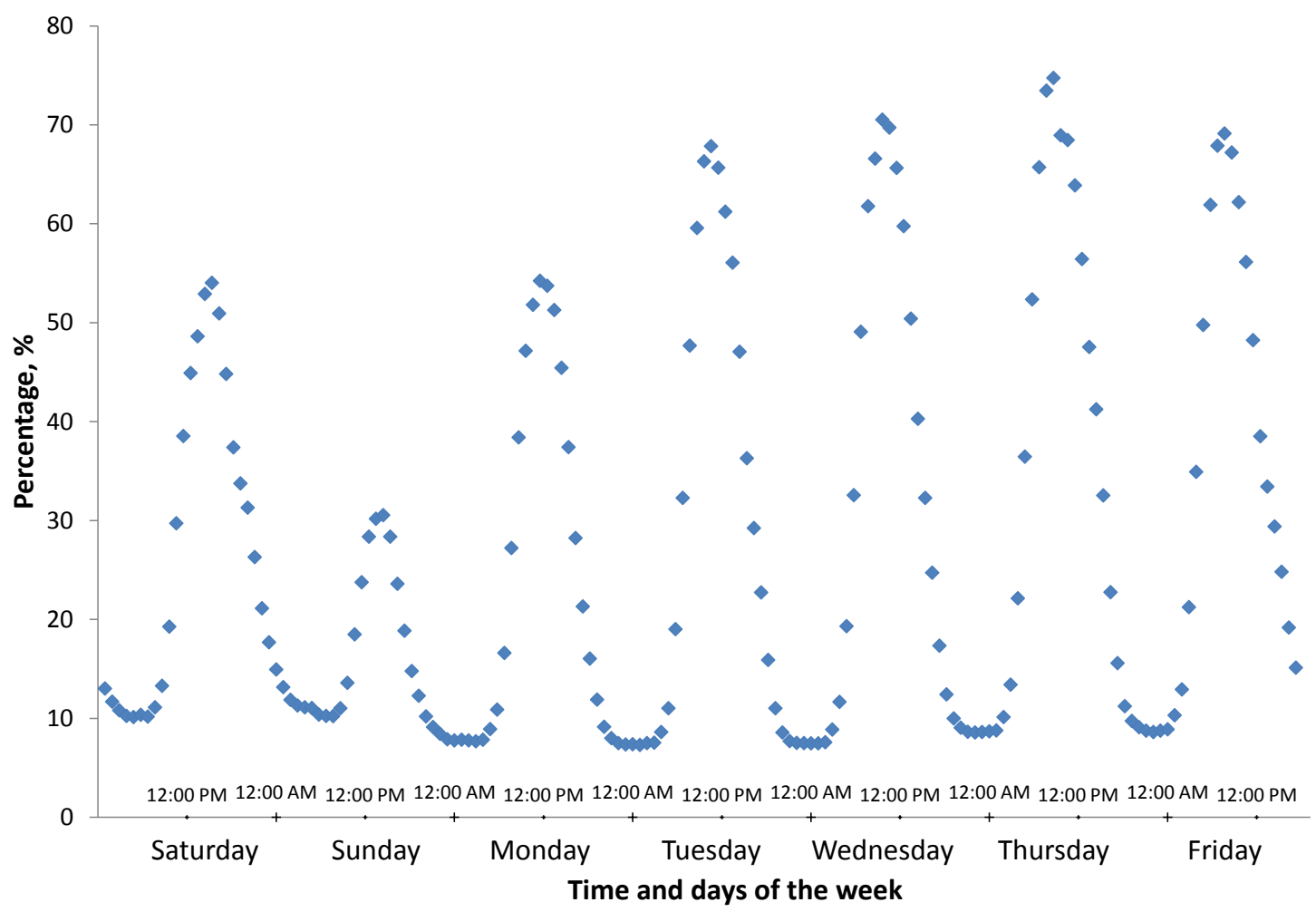

Fig. 6. Sutter-Stockton parking garage distribution in different days of the week.

\section{Accumulated peak rate of heat release}

For each simulation run, the model specifies the location of each vehicle in the parking area and the class of the vehicle, from which the peak heat release rate of each vehicle can be identified. A single iteration of the simulation will select vehicle classes based on the vehicle classification probability distribution. Thus, every single iteration will produce a different accumulated peak heat release rate. By executing a sufficiently large FIRE SAFETY SCIENCE-PROCEEDINGS OF THE ELEVENTH INTERNATIONAL SYMPOSIUM pp. 944-957 
number of iterations, a range of possible scenarios is obtained. To simplify the analysis, these ranges of peak heat release rate for a given number of vehicles are averaged.

The accumulated peak heat release rate for vehicles are recorded from each simulation iteration. Trial runs of 10,000 iterations for single vehicle fire up to 11 simultaneous vehicle fires are recorded. This is shown in Fig. 7, where it can be seen that the average peak heat release rate for a single vehicle to 11 vehicles shows a linear fit. In Fig. 7 also shows the range of total accumulated peak heat release rate from the iterations. To verify the linearity assumption, 10,000 iterations is run for the peak heat release rate accumulation for 20 vehicles. Thus, the equation of the linear fit is used in the fire risk analysis to obtain the peak heat release rate for a specified number of vehicles.

It is also noted that the usage of probability distribution in the simulation will produces outlier(s) based on the extreme ends of the distribution shape. This explains why, for example for a single vehicle fire can reach over $40,000 \mathrm{~kW}$.

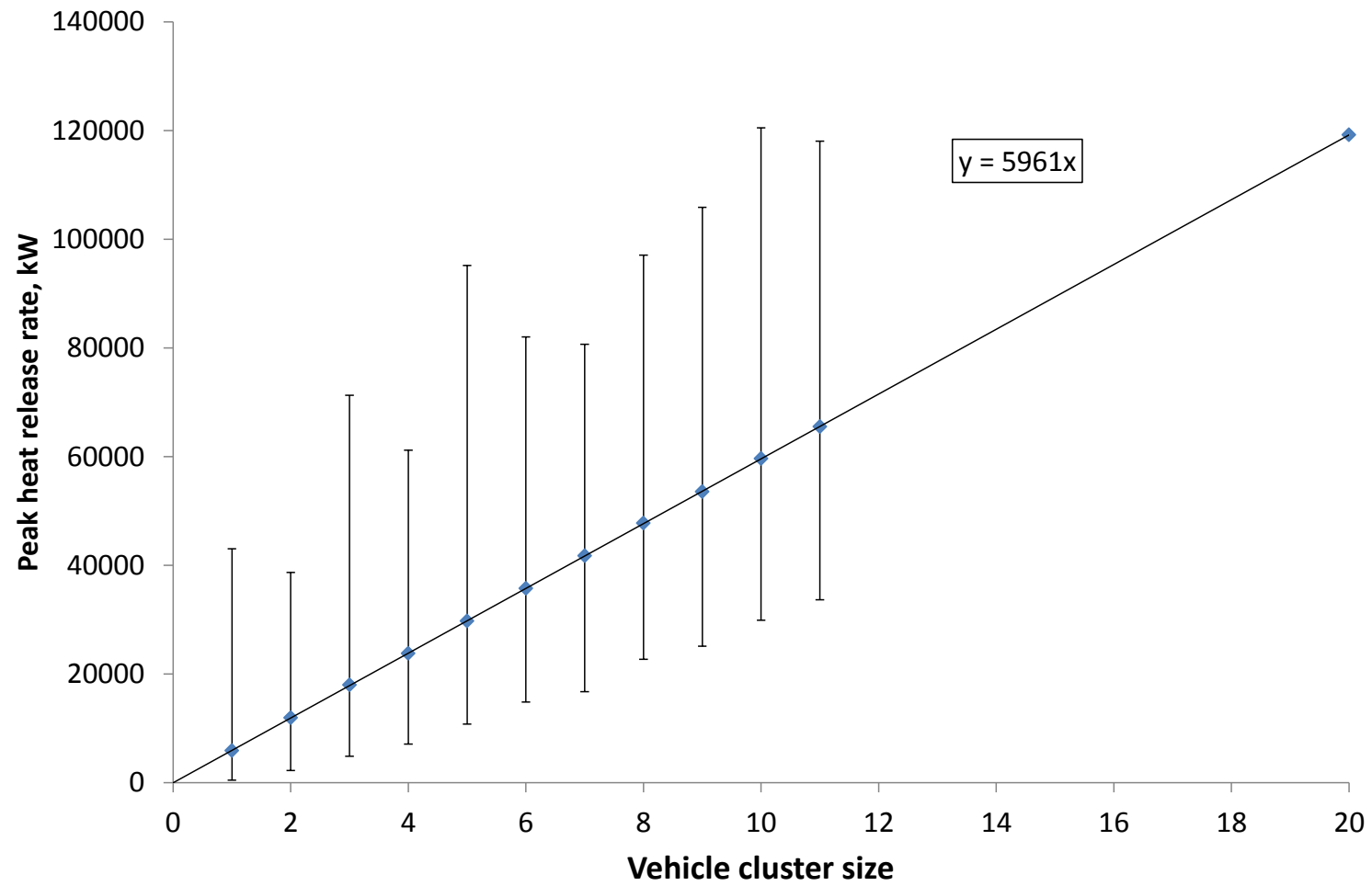

Fig. 7. The total peak heat release rate for increasing vehicle cluster size.

\section{EXAMPLE ANALYSIS}

An example of the application of the fire risk analysis approach is demonstrated with the inputs being 100 parking spaces, 75 vehicles (i.e. a $75 \%$ occupancy) and 10,000 iterations for the parking simulation model. A two-row parking arrangement with a tendency factor weighting of 0.90 and Method 1 for determining fire scenarios is used in this example.

The outcome of the parking simulation model is in the form of the probability of having different cluster sizes, the vehicle involvement probabilities and total rates of heat release. The probability of having different cluster sizes is shown in the second column of Table 6. However, the results are not shown for all 75 vehicle clusters because not every one is obtained through the 10,000 iterations. Thus, only clusters of vehicles with probability results are shown where it can be seen that the highest occurs for the 51-vehicle cluster at 0.174. The second highest probability is the cluster with 52 vehicles at 0.135 . This means that during the iterations, 
the simulation model tends to repeatedly form the 51 or 52 vehicle clusters in preference to other cluster sizes.

The third column in Table 6 is the vehicle involvement probability obtained using Fig 3 and the total rate of heat release for a given cluster size is obtained from Fig. 7. The total heat release rate shows values that can exceed $380 \mathrm{MW}$ which is a manifestation of the assumption that all cars ignite and burn simultaneously. However whether this value could be achieved would also depend on the ventilation available within a particular car park and any modelling would need to account for such conditions.

The fire risk level in Table 6 shows that the highest risk of vehicle fire is for a single vehicle at $4.90 \times$ $10^{-4}$. Even though, the total accumulated heat release rate for a single vehicle is low, the vehicle involvement probability governs the whole fire risk level. This is due to the large difference in the orders of magnitude since the vehicle involvement probability follows a power law.

Table 6. Simulation and fire risk analysis by using Method 1.

\begin{tabular}{|c|c|c|c|c|}
\hline $\begin{array}{l}\text { Number of } \\
\text { vehicles }\end{array}$ & $\begin{array}{l}\text { Cluster size probabil- } \\
\text { ity }\end{array}$ & $\begin{array}{l}\text { Vehicle involvement } \\
\text { probability }\end{array}$ & $\begin{array}{l}\text { Total rate of heat re- } \\
\text { lease }(\mathrm{kW})\end{array}$ & Fire risk level \\
\hline 1 & 0.041 & $2.00 \times 10^{-6}$ & 5952 & $4.90 \times 10^{-4}$ \\
\hline 2 & 0.032 & $3.61 \times 10^{-7}$ & 11913 & $1.36 \times 10^{-4}$ \\
\hline 3 & 0.042 & $1.32 \times 10^{-7}$ & 17874 & $9.94 \times 10^{-5}$ \\
\hline 4 & 0.038 & $6.51 \times 10^{-8}$ & 23835 & $5.94 \times 10^{-5}$ \\
\hline 5 & 0.033 & $3.75 \times 10^{-8}$ & 29796 & $3.65 \times 10^{-5}$ \\
\hline 6 & 0.029 & $2.39 \times 10^{-8}$ & 35757 & $2.50 \times 10^{-5}$ \\
\hline 7 & 0.020 & $1.63 \times 10^{-8}$ & 41718 & $1.36 \times 10^{-5}$ \\
\hline 8 & 0.016 & $1.17 \times 10^{-8}$ & 47680 & $8.95 \times 10^{-6}$ \\
\hline 9 & 0.013 & $8.77 \times 10^{-9}$ & 53641 & $6.21 \times 10^{-6}$ \\
\hline 10 & 0.007 & $6.76 \times 10^{-9}$ & 59602 & $2.79 \times 10^{-6}$ \\
\hline 11 & 0.006 & $5.34 \times 10^{-9}$ & 65563 & $2.26 \times 10^{-6}$ \\
\hline 12 & 0.005 & $4.31 \times 10^{-9}$ & 71524 & $1.48 \times 10^{-6}$ \\
\hline 13 & 0.003 & $3.54 \times 10^{-9}$ & 77485 & $7.60 \times 10^{-7}$ \\
\hline 14 & 0.002 & $2.94 \times 10^{-9}$ & 83446 & $5.50 \times 10^{-7}$ \\
\hline 15 & 0.001 & $2.48 \times 10^{-9}$ & 89407 & $1.78 \times 10^{-7}$ \\
\hline 18 & 0.001 & $1.58 \times 10^{-9}$ & 107291 & $8.15 \times 10^{-8}$ \\
\hline 20 & 0.001 & $1.22 \times 10^{-9}$ & 119213 & $7.75 \times 10^{-8}$ \\
\hline 48 & 0.001 & $1.40 \times 10^{-10}$ & 286124 & $5.13 \times 10^{-8}$ \\
\hline 49 & 0.004 & $1.33 \times 10^{-10}$ & 292085 & $1.53 \times 10^{-7}$ \\
\hline 50 & 0.027 & $1.27 \times 10^{-10}$ & 298046 & $1.01 \times 10^{-6}$ \\
\hline 51 & 0.174 & $1.21 \times 10^{-10}$ & 304007 & $6.39 \times 10^{-6}$ \\
\hline 52 & 0.135 & $1.15 \times 10^{-10}$ & 309968 & $4.80 \times 10^{-6}$ \\
\hline 53 & 0.099 & $1.10 \times 10^{-10}$ & 315929 & $3.43 \times 10^{-6}$ \\
\hline 54 & 0.071 & $1.05 \times 10^{-10}$ & 321890 & $2.38 \times 10^{-6}$ \\
\hline 55 & 0.059 & $1.00 \times 10^{-10}$ & 327851 & $1.93 \times 10^{-6}$ \\
\hline 56 & 0.043 & $9.58 \times 10^{-11}$ & 333812 & $1.38 \times 10^{-6}$ \\
\hline 57 & 0.029 & $9.17 \times 10^{-11}$ & 339773 & $9.00 \times 10^{-7}$ \\
\hline 58 & 0.023 & $8.78 \times 10^{-11}$ & 345735 & $7.04 \times 10^{-7}$ \\
\hline 59 & 0.016 & $8.42 \times 10^{-11}$ & 351696 & $4.66 \times 10^{-7}$ \\
\hline 60 & 0.013 & $8.08 \times 10^{-11}$ & 357657 & $3.70 \times 10^{-7}$ \\
\hline 61 & 0.007 & $7.75 \times 10^{-11}$ & 363618 & $1.83 \times 10^{-7}$ \\
\hline 62 & 0.005 & $7.45 \times 10^{-11}$ & 369579 & $1.37 \times 10^{-7}$ \\
\hline 63 & 0.005 & $7.16 \times 10^{-11}$ & 375540 & $1.36 \times 10^{-7}$ \\
\hline 64 & 0.002 & $6.89 \times 10^{-11}$ & 381501 & $4.48 \times 10^{-8}$ \\
\hline
\end{tabular}


A sensitivity analysis has been carried out by varying the weighting of the tendency factor in the example analysis. In this analysis, the same number of parking spaces and number of vehicles were used while the tendency factor weighting is differed from 0.70 to 0.90 based on the analysis of Waerden et al.'s [5] data.

Fig. 8 shows how the parking tendency factor alters the average parking probability of each multiple vehicle cluster size. It is obvious that as the tendency factor weighting increases it will produce greater probabilities of large vehicle clusters. This sensitivity analysis also considers a random distribution i.e. a tendency factor weighting of $50 \%$ which is shown by the $\times$ symbols. The addition of the random distribution is presented for the purpose of comparison as people invariably have a range of parking behaviour tendencies [14] that would mean it is not a random process. However it is interesting to note that when the distribution is random it produces the highest probability of a single vehicle cases.

Fig. 9 compares the fire risk level for different tendency factor weightings i.e. 0.70, 0.80 and 0.90, and the random distribution. The graph is shown using a semi-log scale to more clearly illustrate the wide range in the results as the cluster size increases. From Fig. 9, varying the tendency factor weighting also affects the fire risk level even though it does not change the fact that a single vehicle fire has the highest fire risk level. This shows the importance of the vehicle involvement probability over the variations in the cluster size probability. The random distribution shows the highest fire risk level for a single vehicle due to the cluster size probability being directly related to the fire risk level.

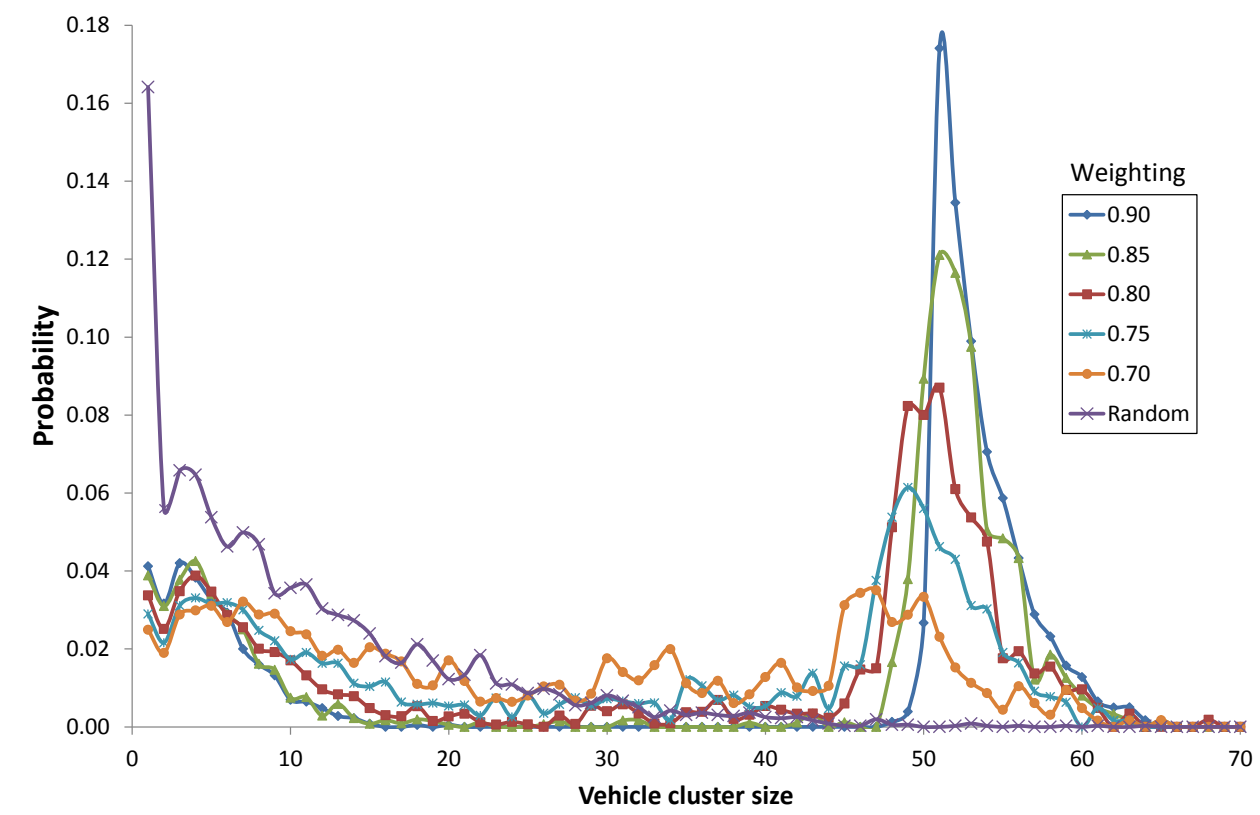

Fig. 8. Cluster size probabilities for 75 vehicles in 100 parking spaces with different parking tendency factor weightings.

\section{CONCLUSION}

There are several limitations upon the fire risk analysis method used in this work. Firstly the online data for parking probability are limited by the range of parking building data available and the distribution of the parked vehicles across the spaces is not included. This limitation could be addressed by making on-site observations in the required car parking building. Secondly the vehicle fire involvement probability used statistics that were a combination of data from different agencies and years. Finally the consequence part was limited due to inadequate rate of heat release data for vehicle experiments that cover Van/MPV, SUV and Passenger car: Heavy classifications. 


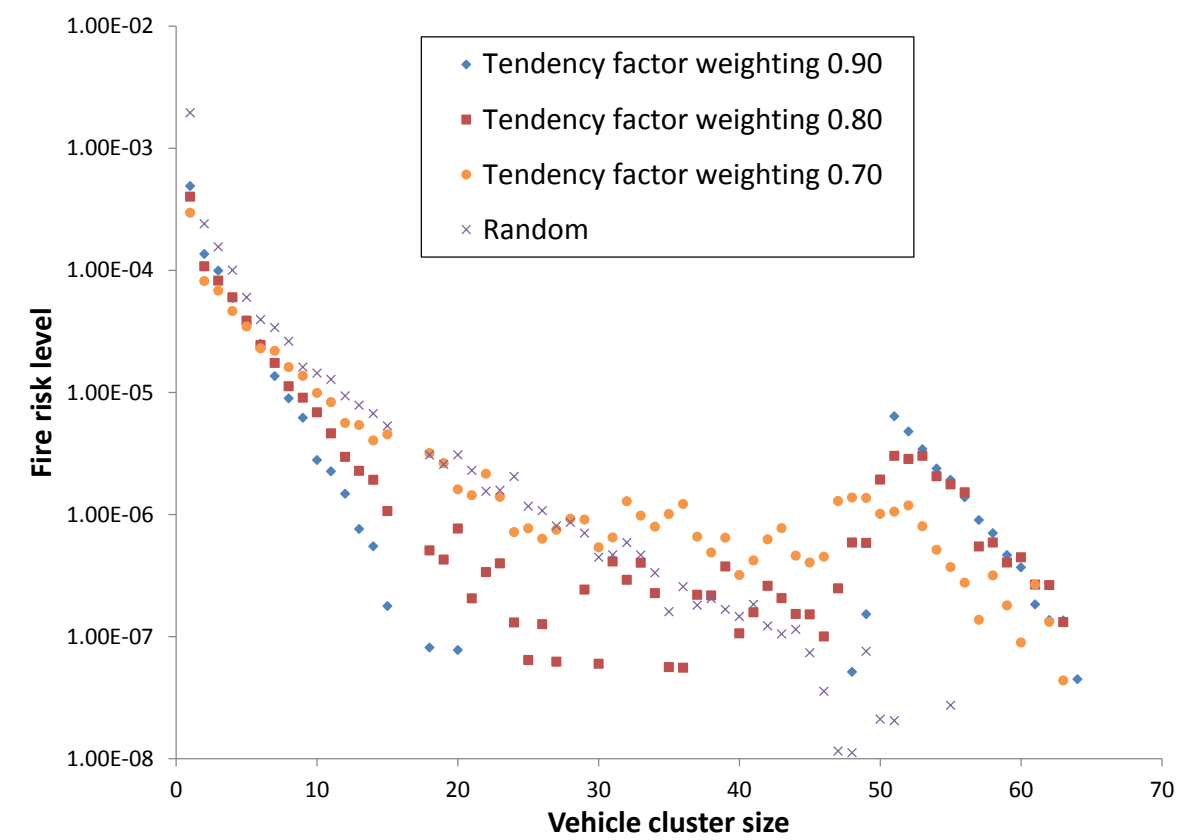

Fig. 9. Fire risk level for 75 vehicles in 100 parking spaces with different parking tendency factor weightings.

By using Method 1 to find the fire scenarios, the highest fire risk is for a single vehicle at $4.90 \times 10^{-4}$ for a $75 \%$ occupancy. More vehicles involved means higher consequences but the vehicle involvement probability governs the whole fire risk analysis since it shows significant difference in the order of magnitude of the probability. Thus, more attention to the collection of vehicle involvement probability is needed in future studies. The next steps in this research are to examine the fire growth characteristics of car fires rather than to only consider the peak rate of heat release and to model the spread of fire between cars using a tool such as B-RISK [15].

It is also noted that the current data for the vehicle involvement probability does not mention whether any suppression systems were operated or at what stage any fire fighters intervention occurred. Had the information regarding the suppression of the fire in the statistics been included, a more realistic analysis is likely to be produced. Furthermore the statistics do not indicate whether there were neighbouring vehicles present in the incident which could have got involved in the fire. These limitations in the statistics have an impact on the ability to provide appropriate data for a risk analysis model.

An initial assessment of Method 2 to find the fire scenarios suggests that it is likely to produce highest risk for a single vehicle due to a greater weight of probability of having a single vehicle fire. It could be argued that the formation of scenarios using Method 2 already incorporates the vehicle involvement probability. This sets grounds for more research to be carried out in the future.

The flexibility of the model allows for future analysis of car parking buildings with different number of spaces, different occupancy numbers and the effect of human vehicle parking behaviour. In trying to achieve the objective of this research it is acknowledged that there is a continued interest in the phenomenon of travelling fires in which a fire in a large space only burns over a limited area at any one time [16]. A car parking building is identified as one type of structure with the potential for travelling fires. However the fire risk analysis approach discussed here does not try to incorporate travelling fires as it requires more work to be done should it be desirable to include this phenomenon. 


\section{REFERENCES}

[1] Cheong, M.K., Spearpoint, M., and Fleischmann, C., (2008) Using Peak Heat Release Rate to Determine the Fire Risk Level of Road Tunnels, Journal of Risk and Reliability 222: 595-604, http://dx.doi.org/10.1243/1748006XJRR169

[2] Shipp, M., "Fire spread in car parks," Technical Report BD2552, Building Research Establishment (BRE), London,United Kingdom, 2010.

[3] Roosefid, M., and Zhao, B., "Fire safety engineering of an open carpark. Example use of ISO TS 24679,"CTICM, Paris, France, 2011.

[4] Watts, J.M., and Hall, J.R.. "Introduction to Fire Risk Analysis," The SFPE Handbook of Fire Protection Engineering ( $4^{\text {rd }}$ ed), DiNenno P.J. (ed.), National Fire Protection Association, 2008.

[5] Waerden, P.V.D., Borgers, A., Timmermans, H., “Travelers Micro-Behavior at Parking Lots: A Model of Parking Choice Behavior," Processing of the $82^{\text {nd }}$ Annual Meeting of the Transportation Research Board, Washington DC, 2007.

[6] Mohd Tohir, M.Z., and Spearpoint, M., (2013) Distribution analysis of the fire severity characteristics of single passenger road vehicles using heat release rate data, Fire Science Reviews 2:5, http://dx.doi.org/10.1186/2193-0414-2-5

[7] Opland, L., "Size classification of passenger cars," Masters Thesis, Chalmers University of Technology, Goteborg, Sweden, 2007.

[8] American National Standard. "Manual on classification of motor vehicle traffic accidents," ANSI D16.1-2007, 2007.

[9] Subramaniam, R., "Passenger vehicle occupant fatality rates by type and size of vehicle,"DOT HS 809 979. Traffic Safety Facts. Research Note, January 2006.

[10] European Union. "Regional transport statistics, ”Eurostat: Regional Yearbook 2012, 2012.

[11] Li, Y., and Spearpoint, M., (2007) Analysis of vehicle fire statistics in New Zealand parking buildings, Fire technology, 43(2): 93 - 106, http://dx.doi.org/10.1007/s10694-006-0004-2

[12] Challands, N., Personal communication, 2012.

[13] Joyeux, D., Kruppa, J., Cajot, L.-G., Schleich, J.-B., de Leur, P.V., and Twilt, L., "Demonstration of Real Fire Tests in Car Parks and High Buildings," CTICM, Paris, France, 2002.

[14] Chen, M., Hu, C., and Chang, T. "The research on optimal parking space choice model in parking lots,'In Computer Research and Development (ICCRD) 2011 3rd International Conference, March 2011, pp 93-97.

[15] Wade, C., Baker, G., Frank, K., Robbins, A., Harrison, R., Spearpoint, M., Fleischmann, C.M., "B-RISK user guide and technical manual,"SR 282. BRANZ Ltd, New Zealand, 2013.

[16] Stern-Gottfried, J., Rein, G., (2012) Travelling fires for structural design Part I: Literature review, Fire Safety Journal 54: 74-85, http://dx.doi.org/10.1016/j.firesaf.2012.06.003 